\title{
Goshajinkigan, a Traditional Japanese Medicine, Suppresses Voltage-Gated Sodium Channel Nav1.4 Currents in C2C12 Cells
}

\author{
Ryota Imai, ${ }^{1}$ Shoichiro Horita, ${ }^{1}$ Yuko Ono, ${ }^{1,2}$ Keisuke Hagihara, ${ }^{3}$ Masaru Shimizu, ${ }^{1,4}$ \\ Yuko Maejima, ${ }^{1}$ and Kenju Shimomura ${ }^{1, *}$
}

\begin{abstract}
Goshajinkigan (GJG) is a traditional Japanese Kampo medicine used clinically to treat muscle pain in Japan. However, its underlying mechanism remains unclear. Since voltage-gated sodium channel (Nav) 1.4 is involved in skeletal muscle contraction, we investigated the possibility that GJG may affect Nav1.4 currents. By using an electrophysiological technique on skeletal muscle cell line C2C12, we found that GJG suppresses Nav1.4 currents in C2C12 cells. It is suggested that GJG may improve skeletal muscle stiffness or cramps by inhibiting abnormal Nav1.4 excitation. GJG may act as a Nav1.4 blocker and may be useful to treat muscle stiffness and clamps as well as easing the pain.
\end{abstract}

Keywords: C2C12; goshajinkigan; Kampo; skeletal muscle; traditional Japanese medicine; voltage-gated sodium channel

\section{Introduction}

Voltage-gated sodium channels (Nav) are important to initiate and propagate action potential in the excitable tissues such as the skeletal muscle, cardiac muscle, and nerve. Nine subtypes (Nav1.1-Nav1.9) have been identified in mammals with Nav1.4 predominantly expressed in the skeletal muscle. ${ }^{1}$ Nav1.4 is activated by sensing a motor endplate potential change, which eventually causes myofiber excitation. ${ }^{2}$ It is assumed that the excessive activation of Nav1.4 may cause abnormal skeletal muscle contraction. Sodium channel blockers are known to be effective for muscle stiffness in patients with a mutation in the SCN4A gene encoding Nav1.4 and for muscle cramps in amyotrophic lateral sclerosis (ALS) patients. ${ }^{3,4}$

Goshajinkigan (GJG), a traditional Japanese Kampo medicine, has been used clinically in Japan to treat lower back pain, rhigosis or numbness of the extremities, and melosalgia. GJG is also reported to improve chemotherapy-induced and diabetic neuropathy. ${ }^{5,6}$ However, recently, the effects of GJG on the skeletal muscle have been focused. ${ }^{7-9}$ Some clinical reports show that GJG relieves pain caused by skeletal muscle cramps. ${ }^{10-12}$ Therefore, GJG may suppress abnormal skeletal muscle excitation and relieve muscle stiffness or cramps.

To date, no reports show the effects of GJG on the skeletal muscle excitability. In this study, we investigated whether GJG can suppress Nav currents that regulate muscle excitability of $\mathrm{C} 2 \mathrm{C} 12$ cells.

\section{Materials and Methods}

Cell culture

Cell culture was performed according to the previous report. ${ }^{13}$ Murine C2C12 myoblasts (RIKEN Cell Bank,

\footnotetext{
${ }^{1}$ Department of Bioregulation and Pharmacological Medicine, Fukushima Medical University School of Medicine, Fukushima, Japan.

${ }^{2}$ Department of Disaster and Emergency Medicine, Graduate School of Medicine, Kobe University, Kobe, Japan.

${ }^{3}$ Department of Kampo Medicine, Osaka University Graduate School of Medicine, Osaka, Japan.

${ }^{4}$ Department of Neurology, Matsumura General Hospital, Fukushima, Japan.
}

*Address correspondence to: Kenju Shimomura, MD, PhD, Department of Bioregulation and Pharmacological Medicine, Fukushima Medical University, 1 Hikarigaoka, Fukushima, 960-1247, Japan, E-mail: shimomur@fmu.ac.jp

(c) Ryota Imai et al. 2020; Published by Mary Ann Liebert, Inc. This Open Access article is distributed under the terms of the Creative Commons License (http://creativecommons.org/licenses/by/4.0), which permits unrestricted use, distribution, and reproduction in any medium, provided the original work is properly cited. 
Ibaraki, Japan) were cultured in high-glucose Dulbecco's modified Eagle's medium (DMEM) supplemented with $10 \%(\mathrm{v} / \mathrm{v})$ fetal bovine serum, $100 \mathrm{U} / \mathrm{mL}$ penicillin, and $100 \mu \mathrm{g} / \mathrm{mL}$ streptomycin at $37^{\circ} \mathrm{C}$ in a humidified atmosphere containing $5 \% \mathrm{CO}_{2}$. After the cells reached confluence, the culture medium was changed to highglucose DMEM supplemented with $2 \%(\mathrm{v} / \mathrm{v})$ heatinactivated horse serum, $100 \mathrm{U} / \mathrm{mL}$ penicillin, and $100 \mu \mathrm{g} / \mathrm{mL}$ streptomycin to induce myogenic differentiation. C2C12 cells on day 5-13 after differentiation were used in experiments.

\section{Reagents}

GJG was obtained from Tsumura and Co. (Tokyo, Japan), manufactured by spray-drying a hot water extract of a mixture of 10 crude drugs: Rehmanniae radix (5g), Achyranthis radix (3 g), Corni fructus (3 g), Moutan cortex (3 g), Alismatics rhizome (3g), Dioscoreae rhizome $(3 \mathrm{~g})$, Plantaginis semen (3g), Hoelen (3 g), processed Aconiti tuber (1 g), and Cinnamomi cortex (1 g). GJG $(50,100,500 \mu \mathrm{g} / \mathrm{mL})$ was suspended in an extracellular solution, then centrifuged $(5000 \mathrm{rpm}, \sim 3000 \times g)$ for $10 \mathrm{~min}$ and used after filtering $(0.22 \mu \mathrm{m})$. Tetrodotoxin (TTX, $100 \mathrm{nM}$ ) was dissolved in an extracellular solution.

\section{Electrophysiology}

Whole-cell patch-clamp recordings were performed referring to the previous report. ${ }^{14}$ Spindle cells were selected for the electrophysiological experiments. All recordings were performed in voltage-clamp mode at room temperature $\left(22-25^{\circ} \mathrm{C}\right)$ using an EPC-800 patchclamp amplifier (HEKA Electronics, Lambrecht/Pfalz, Germany) filtered at $1 \mathrm{kHz}$. Data were digitized with an analog-to-digital converter (Molecular Devices, CA) and stored on a computer using Clampex 10.5 software (Molecular Devices). Patch electrodes (3-6 M $)$ ) were filled with an internal solution containing (in $\mathrm{mM}$ ) 105 CsF, $10 \mathrm{NaCl}, 10$ ethylene glycol tetraacetic acid, and 10 2-[4-(2-hydroxyethyl)-1-piperazinyl]ethanesulfonic acid (HEPES) (pH 7.3 with $\mathrm{CsOH}$ ). The extracellular solution contained (in $\mathrm{mM}$ ) $140 \mathrm{NaCl}, 2.5 \mathrm{KCl}, 1$ $\mathrm{MgCl}_{2}, 1 \mathrm{CaCl}_{2}$, and 10 HEPES (pH 7.4 with $\mathrm{NaOH}$ ). Data were analyzed using Clampfit 10.5 software (Molecular Devices). For the measurement of Nav currents, the cells were held at $-120 \mathrm{mV}$ and the currents were evoked by $20 \mathrm{msec}$ voltage depolarization to voltage values between -80 and $+50 \mathrm{mV}$ in $10 \mathrm{mV}$ increments. GJG or TTX was applied to the cells through bath perfusion. The currents were recorded 3-10 min after exposure to these drugs or washout. GJG concen- tration-response curve of Nav was fitted with the following equation: $\mathrm{G} / \mathrm{Gc}=a+(1-a) /\left(1+\left([\mathrm{GJG}] / \mathrm{IC}_{50}\right)^{h}\right)$, where [GJG] is the GJG concentration, $a$ is the fraction of unblocked current at saturating [GJG], $\mathrm{IC}_{50}$ is the [GJG] at which inhibition is half-maximal, and $h$ is the slope factor (Hill coefficient). To control for possible rundown, Gc was taken as the mean of the conductance in control solution before and after GJG application. The mean \pm standard error of the mean (SEM) values of $\mathrm{IC}_{50}$ and $h$ were calculated by fitting each individual dose-response curve and calculating the mean of the values obtained.

\section{Quantitative reverse transcription-polymerase} chain reaction

Total RNA from $\mathrm{C} 2 \mathrm{C} 12$ cells after differentiation induction [by replacing 10\% (v/v) fetal bovine serum with $2 \%(\mathrm{v} / \mathrm{v})$ horse serum] was extracted using ISOGEN (Nippon Gene, Tokyo, Japan) according to the manufacturer's instructions, which was then followed by quantitative reverse transcriptase-polymerase chain reaction (qRT-PCR) assay. In brief, total amount of $1 \mu \mathrm{g}$ RNA was used for reverse transcription, and the casein kinase 2a2 (csnk2a2) gene was used for normalizing gene expression. The primers used for csnk2a2 and Nav1.4 genes are 5'-GGAGGCCCTAGATCTTCTTG$3^{\prime}$ (forward) $/ 5^{\prime}$-CGCGTTAAGACGTTTTGATT-3' (reverse), and 5'-GCCTTGCGCTCTCTGACTTG-3' (forward) $/ 5^{\prime}$-ACAGCGTGGGTGACACAAAGTA-3' (reverse), respectively.

\section{Statistical analysis}

The statistical analyses were performed by two-way repeated-measures ANOVA with post hoc Bonferroni's test for current-voltage relationship curves. All data were expressed as mean \pm SEM.

\section{Results}

To investigate whether GJG can suppress Nav currents in $\mathrm{C} 2 \mathrm{C} 12$ cells, we measured Nav currents before and after the application of GJG using the whole-cell patch-clamp technique. Figure $1 \mathrm{~A}$ shows a voltage protocol for recording Nav currents. Figure 1B shows representative Nav currents in control solution, GJG solution, and after the washout. Current-voltage relationship curves indicate that GJG significantly suppressed inward Nav currents between -50 and $-20 \mathrm{mV}$ compared with the control group, and that the suppressed currents were not recovered by washout (within $10 \mathrm{~min}$ ) (Fig. 1C). The amplitude of current 


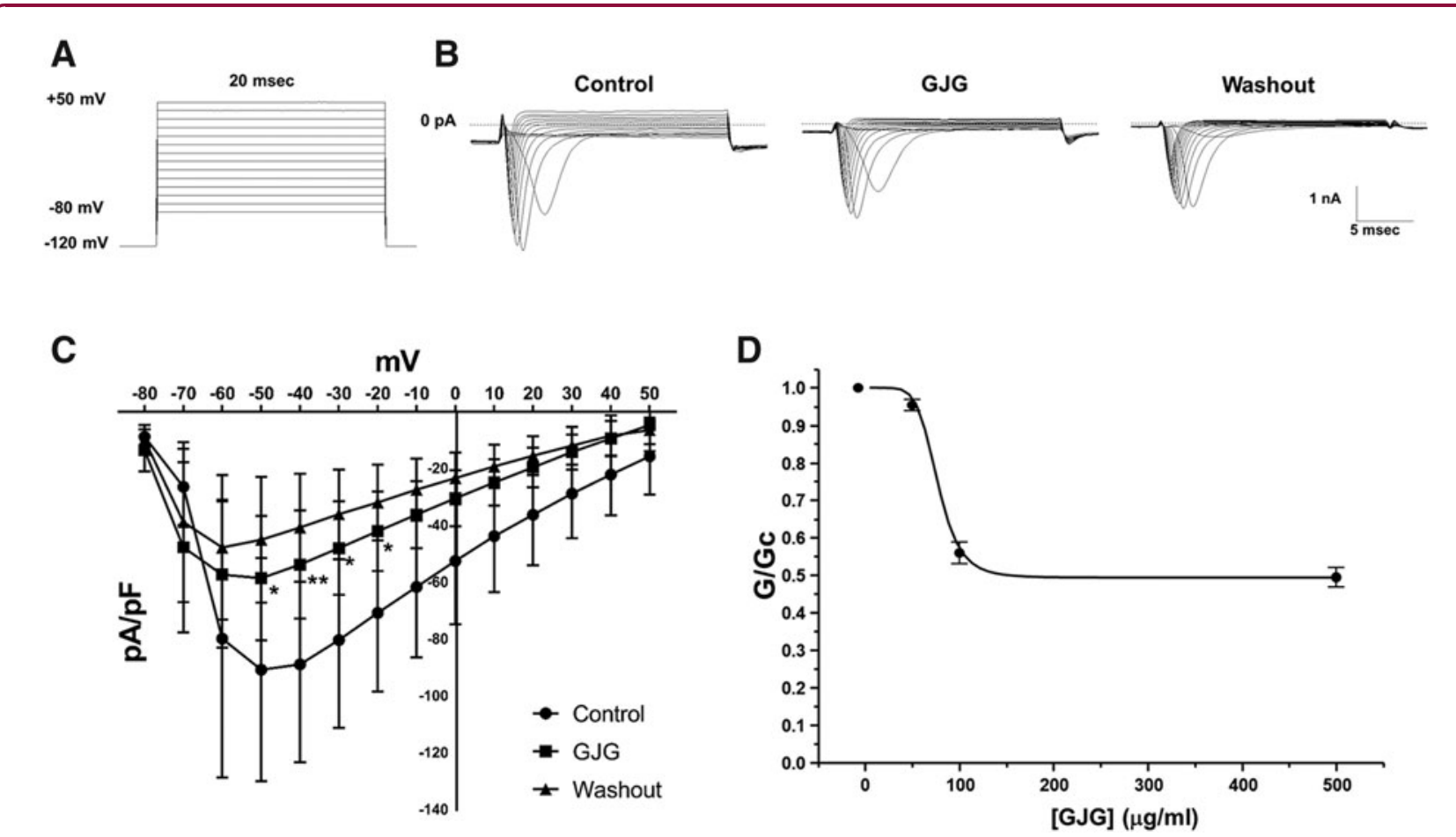

FIG. 1. The effects of goshajinkigan (GJG) on Nav currents of $\mathrm{C} 2 \mathrm{C} 12$ cells. (A) A voltage protocol for evoking Nav currents. (B) The representative Nav currents in control solution, GJG solution $(500 \mu \mathrm{g} / \mathrm{mL})$ and after the washout. (C) Current-voltage relationship curves in control solution, GJG solution $(500 \mu \mathrm{g} / \mathrm{mL})$, and after the washout ( $n=5$, the control group was compared with the GJG group and the washout group by two-way repeated-measures ANOVA post hoc Bonferroni's test, ${ }^{*} p<0.05,{ }^{* *} p<0.01$, control vs. GJG). (D) Mean relationship between GJG concentration and Nav conductance (G), expressed relative to the conductance in the absence of GJG (Gc) for Nav $(n=5)$. The smooth curves are the best fit of the equation to the mean data, with the following parameters: $\mathrm{IC}_{50}=73.13 \mu \mathrm{g} / \mathrm{mL}, h=6.09, a=0.49$. ANOVA, analysis of variance; GJG, goshajinkigan; Nav, voltage-gated sodium channel.

varied depending on the recorded cells. This may be due to the difference in expression rate in each cell. The inhibition by GJG on the peak current in $-50 \mathrm{mV}$ was shown at dose dependency $\left(\mathrm{IC}_{50}=73.13\right.$ $\mu \mathrm{g} / \mathrm{mL}$ ) (Fig. 1D). However, Nav current was incompletely inhibited at even the highest concentration.

We sought to clarify the subtypes of Nav blocked by GJG. Since C2C12 cells are reported to express Nav1.4 and Nav1.5, ${ }^{15}$ we compared the expression level of the genes in our C2C12 cells by qRT-PCR. The result showed that the expression level of Nav1.4 is larger than that of Nav1.5 (data not shown), suggesting that Nav1.4 may contribute to overall Nav current. In addition, our qRT-PCR assay confirmed the expression ratio of Nav1.4 compared with housekeeping gene csnk2a2 was $\sim 27.6$. We distinguished Nav1.4 (TTX- sensitive) currents from Nav1.5 (TTX-resistant) currents by applying $100 \mathrm{nM} \mathrm{TTX}^{1,16}$ and investigated whether GJG can affect the remaining non-Nav1.4 currents after TTX application. Figure 2A shows representative Nav currents in control solution, TTX solution, TTX+GJG solution, and after the washout. Currentvoltage relationship curves indicate that TTX significantly suppressed inward Nav currents between -40 and $\pm 0 \mathrm{mV}$ compared with the control group (Fig. 2B). However, there were no significant differences between the effects of TTX alone and TTX+GJG, indicating that additional GJG application had no effect on the remaining currents after the TTX application. The currents after the TTX and GJG application were not recovered by washout (within $10 \mathrm{~min}$ ). These results suggest that GJG may suppress Nav1.4 but not Nav1.5 in C2C12 cells. 


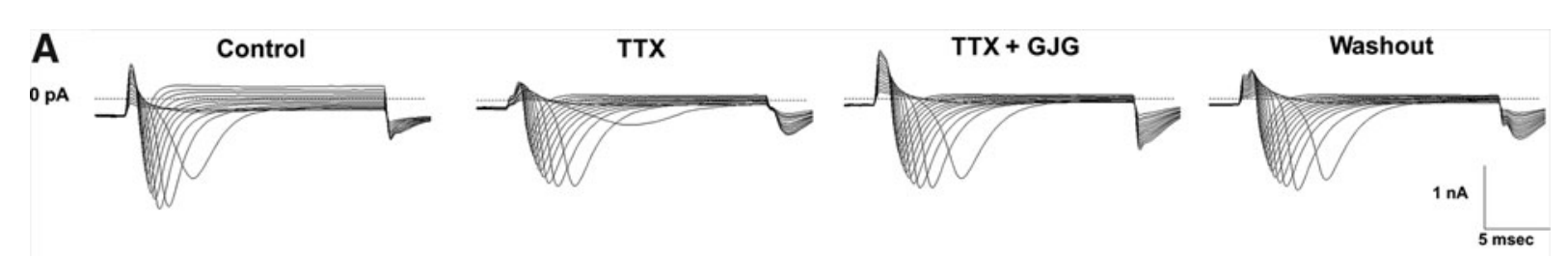

B

$\mathrm{mV}$

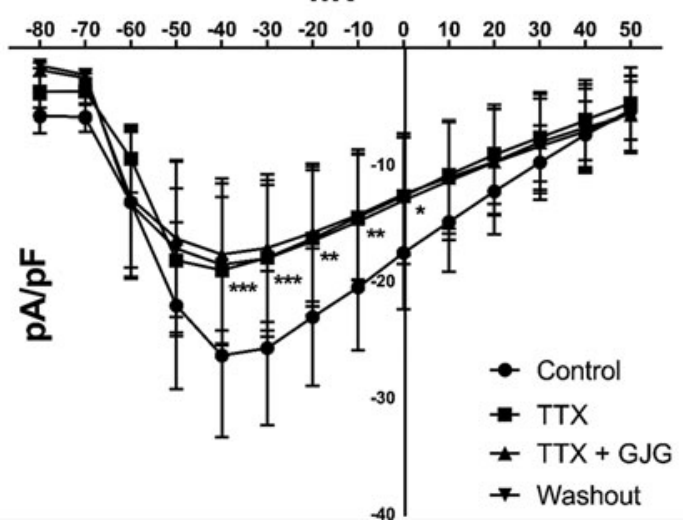

FIG. 2. The effects of goshajinkigan (GJG, $500 \mu \mathrm{g} / \mathrm{mL}$ ) or tetrodotoxin ( $T \mathrm{X}, 100 \mathrm{nM}$ ) on Nav currents of $\mathrm{C} 2 \mathrm{C} 12$ cells. (A) The representative Nav currents in control solution, GJG solution, TTX+GJG solution, and after the washout. (B) Current-voltage relationship curves in control solution, TTX solution, TTX+GJG solution, and after the washout ( $n=6$, the TTX group was compared with the control group, the GJG+TTX group and the washout group by two-way repeated-measures ANOVA post hoc Bonferroni's test, ${ }^{*} p<0.05$, ${ }^{* *} p<0.01,{ }^{* * *} p<0.001$, control vs. TTX). TTX, tetrodotoxin.

\section{Discussion}

Various natural ingredients are known to block Nav. ${ }^{17}$ Since GJG is composed of 10 crude drugs, GJG may contain ingredients that are capable of blocking Nav. Indeed, some ingredients binding to $\mathrm{Nav}$ have been identified in GJG, especially in processed Aconiti tuber. ${ }^{18,19}$ Moreover, it has been reported that lappaconitine or its metabolite $\mathrm{N}$-deacetyllappaconitine, ingredients of processed Aconiti tuber of GJG, can block $\mathrm{Na}$ current of the trigeminal neurons, cardiac muscle cells, or hippocampal pyramidal neurons. ${ }^{20}$ However, Nav1.4 is not expressed in these tissues. To our knowledge, the inhibitory effects of the ingredients of GJG on Nav1.4 have never been studied. Thus, this study may provide a new insight that the ingredients of GJG can block Nav1.4 in the skeletal muscle.

Although, as mentioned earlier, lappaconitine is reported to block $\mathrm{Na}$ current in cardiomyocytes (Nav1.5), ${ }^{20}$ our results showed that GJG may not affect Nav1.5 in C2C12 cells. The reason why GJG failed to inhibit Nav1.5 currents is unclear, but it may be due to the interaction between various ingredients in $\mathrm{GJG}^{18,19}$ or the difference of the tissue used in experiments. Further studies for the effects of GJG on Nav1.5 and the effects of each ingredient on Nav current are required.

Some analgesic mechanisms of GJG for neuropathic pain have been reported. ${ }^{21}$ It is assumed that the analgesic effects of GJG are mainly exerted by functional modification in the central nervous system. ${ }^{22}$ However, the previous reports suggest that GJG may decrease cold hypersensitivity through peripheral TRPA1 and TRPM8 inhibition, and that lappaconitine, 6-benzoylheteratisine, 1-benzoylnapelline, and 14-benzoyltalatisamine, ingredients of processed Aconiti tuber, may exert analgesic effects through the peripheral Nav inhibition..$^{18,19,23}$ Therefore, it is suggested that GJG ingredients may reach and affect the peripheral tissue, including skeletal muscle.

Antiarrhythmic medicines such as mexiletine, which inhibit inward Na current, are effective for treating patients with the Nav1.4 gene mutation and ALS patients 
to prevent skeletal muscle stiffness and cramps. ${ }^{3,4}$ However, antiarrhythmic medicines often show adverse effects and insufficient efficacy. ${ }^{24}$ Therefore, we suggest that GJG may be useful as a complementary medicine for muscle stiffness and cramps caused by Nav1.4 dysfunction.

\section{Conclusion}

Although further clinical studies are required, we suggest that GJG may become a therapeutic medicine for the skeletal muscle stiffness or cramps through blocking Nav1.4.

\section{Authors' Contributions}

K.S. contributed to the overall supervision of this study. R.I. and S.H. conducted experiments and collected the data with support from K.S., Y.M., M.S., Y.O., and R.I. drafted the initial article. K.S., K.H., S.H., and Y.M. critically reviewed and provided important intellectual information for the article. All authors read and approved the final article.

\section{Author Disclosure Statement}

R.I. is an employee of Tsumura \& Co. For all other authors, no competing financial interests exist.

\section{Funding Information}

This study was supported by a Grant-in-Aid for Scientific Research (C) (26461366 for K.S., 15K09395 for Y.M.) from Japan Society for the Promotion of Science (JSPS).

\section{References}

1. Savio-Galimberti E, Gollob MH, Darbar D. Voltage-gated sodium channels: biophysics, pharmacology, and related channelopathies. Front Pharmacol. 2012;3:124.

2. Jurkat-Rott K, Lehmann-Horn F. Muscle channelopathies and critical points in functional and genetic studies. J Clin Invest. 2005;115:20002009.

3. Simkin D, Bendahhou S. Skeletal muscle $\mathrm{Na}^{+}$channel disorders. Front Pharmacol. 2011;2:63.

4. Oskarsson B, Moore D, Mozaffar T, et al. Mexiletine for muscle cramps in amyotrophic lateral sclerosis: a randomized, double-blind crossover trial. Muscle Nerve. 2018;58:42-48.

5. Kono T, Hata T, Morita S, et al. Goshajinkigan oxaliplatin neurotoxicity evaluation (GONE): a phase 2, multicenter, randomized, double-blind, placebo-controlled trial of goshajinkigan to prevent oxaliplatin-induced neuropathy. Cancer Chemother Pharmacol. 2013;72:1283-1290.

6. Watanabe K, Shimada A, Miyaki K, et al. Long-term effects of goshajinkigan in prevention of diabetic complications: a randomized open-labeled clinical trial. Evid Based Complement Alternat Med. 2014;2014:128726

7. Qin B, Nagasaki $M$, Ren $M$, et al. Gosha-jinki-gan (a herbal complex) corrects abnormal insulin signaling. Evid Based Complement Alternat Med. 2004;1:269-276.

8. Kishida Y, Kagawa S, Arimitsu J, et al. Go-sha-jinki-Gan (GJG), a traditional Japanese herbal medicine, protects against sarcopenia in senescenceaccelerated mice. Phytomedicine. 2015;22:16-22.

9. Takemoto Y, Inaba S, Zhang L, et al. An herbal medicine, Go-sha-jinki-gan (GJG), increases muscle weight in severe muscle dystrophy model mice. Clin Nutr Exp. 2017;16:13-23.
10. Motoo $Y$, Taga $H$, Yamaguchi $Y$, et al. Effect of niuche-shen-qi-wan on painful muscle cramps in patients with liver cirrhosis: a preliminary report. Am J Chin Med. 1997;25:97-102.

11. Nishizawa $Y$, Nishizawa $Y$, Amenomori $Y$, et al. A randomized paralleled group comparison in multicenter cooperation: analgesic effect and safety with Gosha-jjinki-gan and Shakuyaku-kanzo-to in the treatment of painful muscle cramps in patients with cirrhosis. Pain Kampo Med. 2000;10: 13-18.

12. Arakawa C, Nitta Y, Ajihara T, et al. A case of unresectable pancreatic cancer successfully treated with Gosha-jinki-gan for muscle cramps related to administration of S-1. Ann Cancer Res Ther. 2017;25:1-4.

13. Ono $Y$, Sakamoto K. Lipopolysaccharide inhibits myogenic differentiation of $\mathrm{C} 2 \mathrm{C} 12$ myoblasts through the Toll-like receptor 4-nuclear factor- $\kappa \mathrm{B}$ signaling pathway and myoblast-derived tumor necrosis factor- $\alpha$. PLoS One. 2017:12:e0182040.

14. Zebedin E, Mille $M$, Speiser $M$, et al. C2C12 skeletal muscle cells adopt cardiac-like sodium current properties in a cardiac cell environment. Am J Physiol Heart Circ Physiol. 2007;292:H439-450.

15. Baroni D, Moran O. Molecular differential expression of voltage-gated sodium channel $\alpha$ and $\beta$ subunit mRNAs in five different mammalian cell lines. J Bioenerg Biomembr. 2011;43:729-738.

16. Trimmer JS, Cooperman SS, Tomiko SA, et al. Primary structure and functional expression of a mammalian skeletal muscle sodium channel. Neuron. 1989;3:33-49.

17. de Lera Ruiz M, Kraus RL. Voltage-gated sodium channels: structure, function, pharmacology, and clinical indications. J Med Chem. 2015;58: 7093-7118.

18. Friese J, Gleitz J, Gutser UT, et al. Aconitum sp. alkaloids: the modulation of voltage-dependent $\mathrm{Na}^{+}$channels, toxicity and antinociceptive properties. Eur J Pharmacol. 1997;337:165-174.

19. Gutser UT, Friese J, Heubach JF, et al., Mode of antinociceptive and toxic action of alkaloids of Aconitum spec. Naunyn Schmiedebergs Arch Pharmacol. 1998;357:39-48.

20. Ameri A. The effects of Aconitum alkaloids on the central nervous system. Prog Neurobiol. 1998;56:211-235.

21. Cascella M, Muzio MR. Potential application of the Kampo Medicine Goshajinkigan for prevention of chemotherapy-induced peripheral neuropathy. J Integr Med. 2017;15:77-87.

22. Suzuki Y, Goto K, Ishige A, et al. Antinociceptive effect of Gosha-jinki-gan, a Kampo medicine, in streptozotocin-induced diabetic mice. Jpn J Pharmacol. 1999;79:169-175.

23. Mizuno K, Kono T, Suzuki Y, et al. Goshajinkigan, a traditional Japanese medicine, prevents oxaliplatin-induced acute peripheral neuropathy by suppressing functional alteration of TRP channels in rat. J Pharmacol Sci. 2014;125:91-98.

24. Matthews E, Hanna MG. Repurposing of sodium channel antagonists as potential new anti-myotonic drugs. Exp Neurol. 2014;261: 812-815.

Cite this article as: Imai $R$, Horita $S$, Ono $Y$, Hagihara $K$, Shimizu M Maejima Y, Shimomura K (2020) Goshajinkigan, a traditional Japanese medicine, suppresses voltage-gated sodium channel Nav1.4 currents in C2C12 cells, BioResearch Open Access 9:1, 116-120, DOI: 10.1089/ biores.2019.0034.

Abbreviations Used
$\mathrm{ALS}=$ amyotrophic lateral sclerosis
$\operatorname{csnk2a2}=$ casein kinase $2 \mathrm{a} 2$
$\mathrm{DMEM}=$ Dulbecco's modified Eagle's medium
$\mathrm{GJG}=$ goshajinkigan
$\mathrm{Nav}=$ voltage-gated sodium channel
$\mathrm{qRT}-\mathrm{PCR}=$ quantitative reverse transcriptase polymerase
chain reaction
$\mathrm{TTX}=$ tetrodotoxin

